\title{
A New Formulation of the Filter-Error Method for Aerodynamic Parameter Estimation in Turbulence
}

\author{
Jared A. Grauer* and Eugene A. Morelli ${ }^{\dagger}$ \\ NASA Langley Research Center, Hampton, Virginia 23681
}

\begin{abstract}
A new formulation of the filter-error method for estimating aerodynamic parameters in nonlinear aircraft dynamic models during turbulence was developed and demonstrated. The approach uses an estimate of the measurement noise covariance to identify the model parameters, their uncertainties, and the process noise covariance, in a relaxation method analogous to the output-error method. Prior information on the model parameters and uncertainties can be supplied, and a post-estimation correction to the uncertainty was included to account for colored residuals not considered in the theory. No tuning parameters, needing adjustment by the analyst, are used in the estimation. The method was demonstrated in simulation using the NASA Generic Transport Model, then applied to the subscale T-2 jet-engine transport aircraft in flight. Modeling results in different levels of turbulence were compared with results from time-domain output error and frequencydomain equation error methods to demonstrate the effectiveness of the approach.
\end{abstract}

\section{Nomenclature}

$\begin{array}{ll}a_{z} & \text { normal acceleration }[\mathrm{g}] \\ C_{L} & \text { lift force coefficient } \\ C_{m} & \text { pitching moment coefficient } \\ \bar{c} & \text { mean aerodynamic chord }[\mathrm{ft}] \\ E[.] & \text { expectation operator } \\ g & \text { gravitational acceleration }\left[\mathrm{ft} / \mathrm{s}^{2}\right] \\ I_{y y} & \text { pitch moment of inertia }\left[\mathrm{slug} \cdot \mathrm{ft}^{2}\right] \\ m & \text { mass [slug] } \\ N & \text { number of samples } \\ n_{x} & \text { number of states } \\ n_{y} & \text { number of outputs } \\ n_{\theta} & \text { number of parameters } \\ q & \text { body-axis pitch rate }[\mathrm{rad} / \mathrm{s}] \\ \bar{q} & \text { dynamic pressure }\left[\mathrm{lbf} / \mathrm{ft}^{2}\right] \\ R^{2} & \text { coefficient of determination } \\ S & \text { wing reference area }\left[\mathrm{ft}{ }^{2}\right] \\ t & \text { time }[\mathrm{s}]\end{array}$

$\begin{array}{ll}V & \text { true airspeed }[\mathrm{ft} / \mathrm{s}] \\ \alpha & \text { angle of attack }[\mathrm{rad}] \\ \Delta & \text { increment } \\ \delta_{e} & \text { elevator deflection }[\mathrm{rad}] \\ \delta_{t} & \text { throttle } \\ \sigma & \text { standard deviation }\end{array}$

Subscripts

$0 \quad$ starting value

\begin{tabular}{ll}
\multicolumn{2}{l}{ Superscripts } \\
-1 & inverse \\
$T$ & transpose \\
$\cdot$ & time derivative \\
$\wedge$ & estimated value \\
$|\cdot|$ & determinant
\end{tabular}

${ }^{*}$ Research Engineer, Dynamic Systems and Control Branch, MS 308. Senior Member AIAA.

${ }^{\dagger}$ Research Engineer, Dynamic Systems and Control Branch, MS 308. Associate Fellow AIAA. 


\section{Introduction}

IRCRAFT parameter estimation consists of determining the values of unknown parameters in a known Amodel structure to achieve the best match of model output to measured output data. For example, a common task is to estimate stability and control derivatives from small-perturbation maneuver data about a nominal flight condition. Maximum-likelihood estimation has evolved as a widely-accepted method for aircraft parameter estimation because of its superior theoretical properties and proven practicality. ${ }^{1,2}$ Maximum-likelihood estimators are divided into three categories according to their treatment of stochastic noise: output error accounts for measurement noise only, equation error accounts for process noise only, and filter error accounts for both process noise and measurement noise.

Output error and equation error methods have had widespread and successful application to a variety of aircraft modeling problems, ${ }^{3,4}$ whereas filter error has been seldom used, and with much more difficulty. One reason for this is that the former methods are considerably simpler than the latter, leading to easier implementation, lower computational costs, and a more straightforward verification of the modeling results. Also, techniques such as data smoothing and using frequency-domain data have been developed to improve and extend the applicability of first two methods. ${ }^{1,5}$ In contrast, filter error can need higher levels of information content in the data and longer computation time, incur convergence and identifiability problems, require significant analyst judgment, produce inaccurate error measures, and mask some modeling problems by fitting the data too well.

There are, however, occasions when both process and measurement noise manifest in the flight data and filter error becomes attractive or necessary. One such instance is flight in atmospheric turbulence. Because turbulence is difficult to measure and complex to model, and can significantly affect the motion of the aircraft, it is often modeled as stochastic process noise in aircraft flight dynamics, which makes the aircraft states stochastic quantities. Because including process noise considerably complicates the analysis, it is standard practice to instead perform flight tests only in calm air. This strategy can, however, lead to project schedule delays and increased costs when inclement weather is encountered. Some aircraft configurations and flight regimes are particularly susceptible to the effects of turbulence. Subscale aircraft, which are now commonly used for flight testing, have higher gust sensitives due to lower mass and inertia. Flexible aircraft, which are becoming more common because of associated fuel savings, have lightly-damped structural resonances that are easily excited by turbulence. Upset conditions, loss of control scenarios, and accidents are also often accompanied by turbulence. In such cases, filter error is an appropriate maximum-likelihood parameter estimation method because it accurately reflects the physics.

This work presents a new formulation of the filter-error algorithm that can be used with general nonlinear dynamical systems. One strength of the proposed formulation is that it closely parallels the standard implementation of the output-error algorithm, which has been successfully applied to a large number of aircraft and flight maneuvers, ${ }^{6}$ and is thus familiar to use. The new formulation of the filter-error method employs a relaxation technique to alternate between estimating unknown model parameters using GaussNewton optimization, and estimating the process noise covariance matrix using state equation innovations, until convergence is achieved. The measurement noise covariance, which has a clear physical interpretation, can be readily obtained using various methods to decrease the burden placed on the estimator. This formulation results in a relatively small number of unknowns, and exhibits accurate parameter estimation without convergence or identifiability issues. No tuning parameters, needing adjustment by the analyst for each situation, are included in this method. The method may therefore be applied in a straightforward and procedural manner to estimation problems.

The paper is organized as follows. Section II poses the estimation problem for nonlinear dynamical systems considering both process noise and measurement noise. Section III places the current work within context of previous work in aircraft parameter estimation in turbulence. The proposed method is explained in Section IV. Section V presents modeling results for three different cases: 1) simulated data in light-tomoderate turbulence, 2) flight test data in light-to-moderate turbulence, and 3) flight test data in severe turbulence. Conclusions are then drawn and discussed in Section VI.

Many of the tools used to generate results shown in this paper are available in the MATLAB ${ }^{\circledR}$ software package called SIDPAC (System IDentification Programs for AirCraft). ${ }^{7,1}$ SIDPAC is bundled with Ref. [1] and is therefore publicly available. The SIDPAC toolbox was developed at NASA Langley Research Center and is continually expanded and improved. 


\section{Problem Description}

This section establishes the mathematical framework for the estimation problem considered. The aircraft flight dynamics are postulated as the nonlinear dynamic system

$$
\begin{aligned}
\dot{\mathbf{x}}(t) & =\mathbf{f}(\mathbf{x}(t), \mathbf{u}(t), \boldsymbol{\theta})+\mathbf{w}(t) \\
\mathbf{y}(t) & =\mathbf{h}(\mathbf{x}(t), \mathbf{u}(t), \boldsymbol{\theta}) \\
\mathbf{z}(i) & =\mathbf{y}(i)+\mathbf{v}(i) \quad i=1,2, \ldots, N
\end{aligned}
$$

where $\mathbf{x}$ is the state, $\mathbf{u}$ is the control input, $\mathbf{y}$ is the model output, $\mathbf{z}$ is the measurement, and $\boldsymbol{\theta}$ is a vector of unknown constant model parameters. Vector functions $\mathbf{f}$ and $\mathbf{h}$ are known. Vectors $\mathbf{w}$ and $\mathbf{v}$ represent additive process noise and measurement noise, which are assumed to be mutually uncorrelated, zero mean, Gaussian white processes with spectral density and covariance $\mathbf{Q}$ and $\mathbf{R}$, respectively, which are defined by

$$
\begin{aligned}
E\left[\mathbf{w}\left(t_{i}\right) \mathbf{w}^{T}\left(t_{j}\right)\right] & =\mathbf{Q}\left(t_{i}\right) \delta\left(t_{i}-t_{j}\right) \\
E\left[\mathbf{v}(i) \mathbf{v}^{T}(j)\right] & =\mathbf{R}(i) \delta_{i j}
\end{aligned}
$$

Equations (1)-(2) are in continuous time and Eq. (3) is in discrete time, resulting in a continuous-discrete formulation of the system which accurately reflects measuring the aircraft outputs using a digital computer.

The system is stochastic because the noise processes are random. Therefore, the initial state is specified by its mean and covariance

$$
\begin{aligned}
\mathbf{x}_{0} & =E[\mathbf{x}(0)] \\
\mathbf{P}_{0} & =E\left[\left(\mathbf{x}(0)-\mathbf{x}_{0}\right)\left(\mathbf{x}(0)-\mathbf{x}_{0}\right)^{T}\right]
\end{aligned}
$$

from which a state estimator is used to predict the resulting state and output history. As aircraft dynamics are routinely simplified to linearized equations, a Kalman filter is often used for state estimation. ${ }^{8}$

Parameter estimation involves determining the values of $\boldsymbol{\theta}$ such that the model output from Eqs. (1)-(2) best matches the measurements in Eq. (3). This can be done by maximizing the likelihood function ${ }^{1}$

$$
\mathcal{L}[\mathbf{z}(i) ; \boldsymbol{\theta}]=(2 \pi)^{-n_{y} / 2}|\mathcal{B}(i)|^{-1 / 2} \cdot \exp \left[-\frac{1}{2} \boldsymbol{v}(i)^{T} \mathcal{B}(i)^{-1} \boldsymbol{v}(i)\right]
$$

where the innovation

$$
\boldsymbol{v}(i)=\mathbf{z}(i)-\mathbf{y}(i)
$$

is the difference between the measured and modeled output, and where $\mathcal{B}$ is the innovation covariance matrix. It is more convenient to minimize the negative logarithm of the likelihood function instead of maximizing the likelihood function directly (note that these optimizations are equivalent because logarithms are monotonic functions), resulting in the optimization cost function

$$
J(\boldsymbol{\theta})=\frac{1}{2} \sum_{i=1}^{N} \boldsymbol{v}(i)^{T} \mathcal{B}(i)^{-1} \boldsymbol{v}(i)+\ln |\mathcal{B}(i)|
$$

The optimal model parameters minimize this cost function, which effectively minimizes the weighted sum of squares and covariance of the innovations, given the model structure and the measured data. 


\section{Parameter Estimation Methods}

This section discusses several methods for aircraft parameter estimation. First is the dual state/parameter estimation problem, followed by the output-error and equation-error methods, and finally the filter-error method.

\section{III.A. Dual State/Parameter Estimation}

The Kalman filter was originally conceived for optimal recursive state estimation for a linear system having Gaussian process and measurement noise. The dual estimation problem was then posed, where both the states and the unknown model parameters are estimated simultaneously. This is mechanized by augmenting the state vector with the model parameters, and appending $\dot{\boldsymbol{\theta}}=\mathbf{0}$ to the dynamic model equations. Because unknown parameters are now states, the dynamic system becomes nonlinear, and a nonlinear version of the Kalman filter must be used. ${ }^{8,9,10}$ However, issues related to tuning the Kalman filter, linearizing the nonlinear dynamic model, and making this method recursive lead to less accurate results than using non-recursive maximum likelihood methods. ${ }^{11}$ Consequently, only parameter estimation in an off-line environment was studied in this work.

\section{III.B. Output Error and Equation Error}

Output error is a maximum-likelihood method, but it is a simplification of the problem stated in Section II, because process noise is neglected. The dynamic model is deterministic because $\mathbf{Q}=\mathbf{0}$, and state estimation reduces to time integration. Gauss-Newton optimization is used to determine the model parameters, and an algebraic equation is used to estimate $\mathbf{R}$. These unknowns are updated in an alternating manner called a relaxation technique, where one is held constant while the other is estimated. There is no proof that relaxation techniques converge, but this method has been applied successfully for many years with excellent results. $^{2,6,1}$ The assumptions employed in output error considerably simplify the estimation problem, which is one reason why output error is routinely used for aircraft parameter estimation. In addition, integration of the state equations is marginally stable with respect to the model parameters, which can lead to strong sensitivities and fast convergence of the estimator, given suitable starting values for the model parameters. The assumptions are also often very realistic because flight tests are usually conducted in calm air and because all sensors contain some measurement noise. Output-error solutions are, however, biased in the presence of process noise, which can result from unmodeled dynamics or turbulence. ${ }^{6,1}$

Equation error is another maximum-likelihood method, but it is also a simplification because measurement noise is neglected. The estimation problem reduces to ordinary least squares, which has a non-iterative and analytical solution. The simplicity and speed of this approach are its strengths. However, this method requires additional techniques to remove noise from the explanatory variables to obtain unbiased solutions, ${ }^{1,12,5}$ and requires that all explanatory variables are either measured or reconstructed for the analysis.

Some authors propose a blending of the parameter estimation methods, to combine strengths and mitigate weaknesses of the individual methods to attain superior results in the presence of both process and measurement noise. In Ref. [13], output error and equation error are combined using a user-selected weighting matrix to attempt to obtain accurate solutions with good convergence properties. Reference [14] describes an interactive program where the user selects feedback gains for the aerodynamic flow angles and body rates to adjust the ratio of process noise to measurement noise in an ad hoc fashion.

Another approach for estimating parameters in turbulence is to treat the effects of turbulence as deterministic, rather than stochastic. In this strategy, additional sensors can be used to measure or estimate the turbulence, which can then be supplied to the estimator as a known input. Reference [15] surveyed commercial sensors for this purpose, and developed methods to account for the effects of turbulence, using upwash factors and time delays in the measurements.

A successful approach to parameter estimation in both calm air and turbulence has been to use equation error in the frequency domain. ${ }^{1,16,17}$ Here, the data is transformed into the frequency domain using a narrow bandwidth encompassing only the dynamics of interest and the excitation. This step effectively removes the measurement noise and avoids other practical problems incurred when using equation error with time-domain data. A recursive formulation of the Fourier transform, combined with the speed of equation error and efficient testing techniques, ${ }^{18}$ has led to successful real-time estimates during flight. However, this 
method will produce increasingly biased parameter estimates as the turbulence level increases relative to the deterministic signal levels. ${ }^{17}$

\section{III.C. Filter Error}

Filter error is the maximum-likelihood estimator that solves the problem described in Section II without further simplification. The complexity of estimating parameters for a nonlinear dynamical system in the presence of unknown levels of process noise and measurement noise, in addition to the standard challenges of model structure determination, uncertainty quantification, and adhering to modeling assumptions, has made the use of filter error for aerodynamic parameter estimation relatively rare. However, there have been advances and successes in applying filter error. As outlined by Maine and Iliff, ${ }^{11}$ these can be categorized into the following three groups.

The first group is called the natural formulation. Because the model parameters and noise covariance matrices are all unknowns, it follows naturally to attempt to estimate $\boldsymbol{\theta}, \mathbf{Q}$, and $\mathbf{R}$ that best match the model outputs to the measured output data. This approach led to high computational costs because of the large number of unknowns and complex interdependencies involved within the Kalman filter. More importantly, convergence issues and numerical singularities for $\mathbf{Q}$ were encountered. As a result, very few practical applications of this formulation have been reported, and these have usually required further compromises. For example, $\mathbf{R}$ was not estimated in Ref. [19], and $\mathbf{Q}$ was not estimated in Ref. [20].

The second group is called the innovation formulation. To circumvent problems associated with estimating noise covariances, $\boldsymbol{\theta}$ and the Kalman gain matrix $\mathbf{K}$ are instead estimated in an indirect manner. When estimates settle, $\mathcal{B}$ is updated using the relaxation technique, and the process repeats until convergence. The innovation formulation eliminated the convergence and numerical computation problems associated with the natural formulation, but introduced new problems. The Kalman gain matrix has $n_{x} \cdot n_{y}$ elements and is typically fully populated, which increases the number of unknowns and can lead to convergence issues. Elements of the Kalman gain have little physical significance, so finding adequate starting values and verifying solutions is difficult. Because the Kalman gain is estimated, a more sophisticated optimization method is needed to constrain values and keep the filter physically realizable.

The third group is the Maine-Iliff formulation. ${ }^{11}$ In this method, $\boldsymbol{\theta}$ and $\mathbf{Q}$ are estimated, followed by $\mathcal{B}$, using the relaxation technique until convergence. This method reduces the computational load, removes the numerical singularities, and mitigates convergence issues that were incurred by the previous formulations. However, this method still requires a sophisticated optimization algorithm to ensure that the solutions correspond to realizable Kalman filters, and intuition is lost by indirectly solving for the unknowns. Of the relatively few implementations of filter error, the Maine-Iliff formulation has been the most popular. ${ }^{21,14,22}$

\section{Proposed Formulation}

\section{IV.A. Method}

Parameter estimation requires an adequate model structure and measured data with sufficient information content. Like output error, filter error poses a nonlinear estimation problem that requires good starting values for the model parameters $\boldsymbol{\theta}_{0}$, and an iterative optimizer. A separate estimate of $\mathbf{R}$ is used in this approach to reduce demands on the estimator, and reliable methods for doing so are discussed later.

Although this formulation is applicable to general nonlinear systems, the problem is now specialized to linear time-invariant systems of the form

$$
\begin{aligned}
\dot{\mathbf{x}}(t) & =\mathbf{A x}(t)+\mathbf{B u}(t)+\mathbf{w}(t) \\
\mathbf{y}(t) & =\mathbf{C x}(t)+\mathbf{D u}(t) \\
\mathbf{z}(i) & =\mathbf{y}(i)+\mathbf{v}(i) \quad i=1,2, \ldots, N
\end{aligned}
$$

because of their common use in aircraft analysis, control, simulation, and system identification, including the results presented later. The matrices contain constant elements that depend on $\boldsymbol{\theta}$ and known quantities.

Similarly, a steady-state Kalman filter is used for state estimation because the noise statistics can be approximated as stationary for short durations of time. Unsteady or nonlinear variants of the Kalman filter can be used instead, without caveat, for other cases. The state covariance update equation reduces, in the steady state, to

$$
\mathbf{A P}+\mathbf{P} \mathbf{A}^{T}-\mathbf{P C}^{T} \mathbf{R}^{-1} \mathbf{C P}^{T}+\mathbf{Q}=\mathbf{0}
$$


which is solved for $\mathbf{P}$. The steady-state Kalman gain is then computed

$$
\mathbf{K}=\mathbf{P C}^{T} \mathbf{R}^{-1}
$$

If no other information is available, $\mathbf{Q}$ is initially set to zero, which corresponds to output error. The Kalman filter has the a priori prediction ${ }^{1,8}$

$$
\dot{\hat{\mathbf{x}}}(t \mid i-1)=\mathbf{A} \hat{\mathbf{x}}(t \mid i-1)+\mathbf{B u}(t)
$$

and the a posteriori measurement update

$$
\hat{\mathbf{x}}(i \mid i)=\hat{\mathbf{x}}(i \mid i-1)+\mathbf{K}[\mathbf{z}(i)-\mathbf{C} \hat{\mathbf{x}}(i \mid i-1)-\mathbf{D u}(i)]
$$

at each time step. The state estimates are used in Eq. (2) or (12) to compute the estimated output.

The innovations are then computed using Eq. (9), and their covariance is estimated as

$$
\hat{\mathcal{B}}=\frac{1}{N} \sum_{i=1}^{N} \boldsymbol{v}(i) \boldsymbol{v}(i)^{T}
$$

which is the maximum likelihood estimate for a fixed $\boldsymbol{\theta}$. Although the Kalman filter also estimates the innovation covariance, Eq. (18) is preferred here because it is independent of the state estimator chosen and because modeling errors in the Kalman filter bias its innovation covariance estimate. Additionally, Eq. (18) produces a single matrix, instead of a time history of that matrix, which reduces computation.

Gauss-Newton optimization is then employed to update the model parameter estimates. This optimization method is used in the output-error algorithm because it converges quickly near the solution and it is computationally tractable. ${ }^{6,1}$ The estimated output sensitivities

$$
\mathbf{S}(i)=\left\{\frac{\partial \hat{y}_{k}(i)}{\partial \theta_{j}}\right\} \quad \text { for } j=1,2, \ldots, n_{\theta} \text { and } k=1,2, \ldots, n_{y}
$$

are numerically computed using central finite differences. Numerical computations are favored here over analytical derivatives for ease in changing the model structure. The cost function gradient and information matrix

$$
\begin{aligned}
\frac{\partial J}{\partial \boldsymbol{\theta}} & =-\sum_{i=1}^{N} \mathbf{S}(i)^{T} \hat{\mathcal{B}}^{-1} \mathbf{S}(i) \\
\mathbf{M} & =\sum_{i=1}^{N} \mathbf{S}(i)^{T} \hat{\mathcal{B}}^{-1} \mathbf{S}(i)
\end{aligned}
$$

are computed and used to update the model parameters as

$$
\hat{\boldsymbol{\theta}}=\hat{\boldsymbol{\theta}}_{0}-\mathbf{M}^{-1} \frac{\partial J}{\partial \boldsymbol{\theta}}
$$

The covariance of this updated parameter estimate is then described by the Cramér-Rao lower bound

$$
\operatorname{cov}(\hat{\boldsymbol{\theta}}) \geq \mathbf{M}^{-1}
$$

This process repeats until changes in the parameter estimates, cost function, and cost function gradient settle within specified threshold values. Then the relaxation technique is employed to hold $\hat{\boldsymbol{\theta}}$ constant and update $\mathbf{Q}$. Although there are many sophisticated methods for estimating the process noise covariance, a simple method is used here. The process noise is reconstructed from Eq. (11) as

$$
\hat{\mathbf{w}}(t)=\dot{\mathbf{x}}(t)-\mathbf{A} \hat{\mathbf{x}}(t)-\mathbf{B u}(t)
$$

which should be zero-mean. The state derivatives can be obtained by smoothly differentiating the measured states. ${ }^{5,1}$ At the expense of convergence speed, unmeasured states can be substituted using estimates from the Kalman filter. The subtracted terms on the right side of Eq. (24) represent the modeled state vector 
derivative, computed using the current model parameter estimates and the state estimates from the Kalman filter. Afterwards, the process noise covariance is estimated as

$$
\hat{\mathbf{Q}}=\frac{1}{N} \sum_{i=1}^{N} \hat{\mathbf{w}}(i) \hat{\mathbf{w}}(i)^{T}
$$

which may be a fully populated matrix. The optimization then continues with the next iteration of model parameter estimation using this updated process noise covariance. Convergence is attained when changes in the model parameters, cost function, cost function gradient, and process noise covariance matrix all settle within specified threshold values.

The formulation described in this section was implemented using MATLAB ${ }^{\circledR}$ software. The code was developed using the SIDPAC output-error program called oe.m as a starting point.

\section{IV.B. Practical Aspects of Implementation}

A few points in the proposed formulation warrant further discussion. The most outstanding is the assumption that the effects of atmospheric turbulence on the aircraft flight dynamics can be approximated by an additive Gaussian white noise process. Turbulence is inherently complex and its characterization remains an active field of research. Furthermore, previous efforts to accurately identify the gust spectrum introduced additional complexity in the estimator and were unsuccessful. ${ }^{23}$ Filter error with process noise assumed to be white Gaussian noise is an approximate engineering solution, albeit with considerable complexity, to the problem under consideration.

The second point pertains to starting the estimation algorithm. Unlike output error, where poor estimates of initial conditions could cause divergence, ${ }^{24}$ state estimation is relatively robust to these errors and initial conditions can be selected as the first measurement, or otherwise reconstructed from those values. The problem of choosing initial covariance of the initial state is avoided here when selecting a steady-state estimator. Good starting values for the model parameters can usually be obtained from an equation-error analysis, wind tunnel tests, computation fluid dynamics, or engineering experience.

The proposed formulation estimates $\boldsymbol{\theta}$ and $\mathbf{Q}$ for a known $\mathbf{R}$. This is advantageous because it lowers the demands on the estimator and requires less information in the measured data. More importantly, it separates the residuals into components that are physical and well-modeled using covariance $\mathbf{R}$, and other components that are approximated using the spectral density $\mathbf{Q}$. The information contributed during this process can lead to more accurate parameter estimates and uncertainties than other simplifications of the maximum likelihood estimator. In output error for example, measurement noise and process noise, as well as any model structure error, are subsumed in $\mathbf{R}$. These error sources are better accounted for using filter error. Many methods can be employed to determine $\mathbf{R}$, including manufacturer data sheets and ground testing. A global method using optimal Fourier smoothing can also be used to accurately estimate noise characteristics from the flight data. ${ }^{12,1}$ Another simple technique is to average the spectral density of a signal over frequencies in which other responses, such as rigid body or structural dynamics, are small. Figure 1 shows an approximation of the power spectral density of an output measurement. In the bands that are free from contributions other than measurement noise, the spectral density can be used to estimate $\mathbf{R}$.

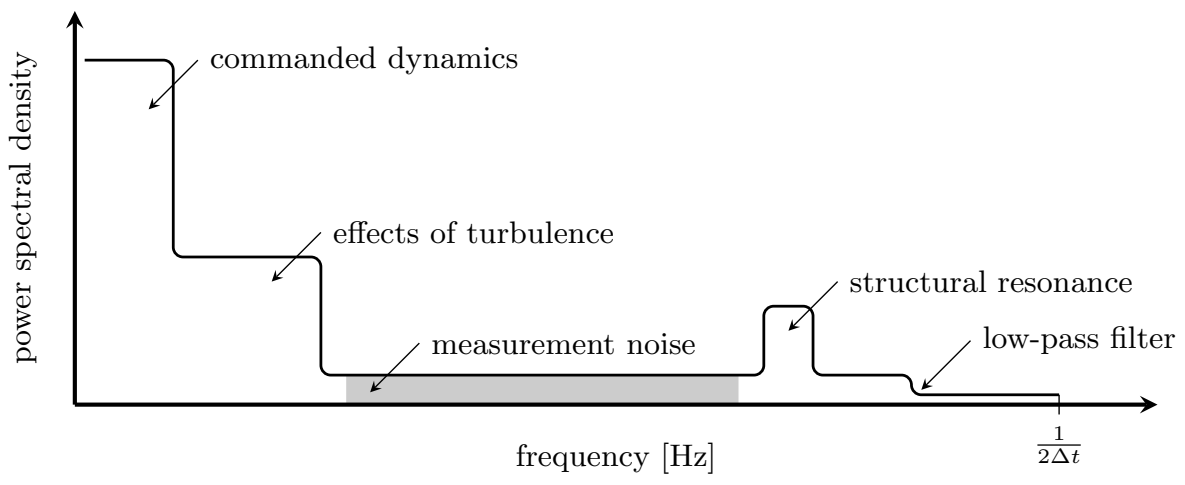

Figure 1. Approximation of power spectral density components 
Another point concerns the estimated output sensitivities, which drive the convergence rate and determine the solution. In output error, state histories are computed via integration, which is very sensitive to changes in the model parameters. In filter error, where a state estimator is used instead, the feedback inherent in the Kalman filter reduces the sensitivity of the model output to the model parameters. While this is beneficial in state estimation applications, in parameter estimation it slows convergence for larger levels of process noise, which is exactly the case being considered. However, it was found that even for flight in severe turbulence, discussed later in Section V, the proposed formulation of the filter error method does not have convergence issues. This is in part due to a more accurate model structure resulting from the new formulation, which better poses the optimization problem and results in fewer local minima.

This formulation of filter error can also incorporate prior information on the model parameters and uncertainty in a Bayesian framework. This is useful when combining results from different analyses, such as wind tunnel tests or a computational fluid dynamics analysis, or from different flight tests and maneuvers. The cost function, cost gradient, information matrix, and Gauss-Newton updates are augmented as

$$
\begin{gathered}
J(\boldsymbol{\theta})=\frac{1}{2} \sum_{i=1}^{N} \boldsymbol{v}(i)^{T} \mathcal{B}^{-1} \boldsymbol{v}(i)+\ln |\mathcal{B}|+\frac{1}{2}\left(\boldsymbol{\theta}-\boldsymbol{\theta}_{p}\right)^{T} \boldsymbol{\Sigma}_{p}^{-1}\left(\boldsymbol{\theta}-\boldsymbol{\theta}_{p}\right) \\
\frac{\partial J}{\partial \boldsymbol{\theta}}=-\sum_{i=1}^{N} \mathbf{S}(i)^{T} \hat{\mathcal{B}}^{-1} \mathbf{S}(i)-\boldsymbol{\Sigma}_{p}^{-1}\left(\boldsymbol{\theta}-\boldsymbol{\theta}_{p}\right) \\
\mathbf{M}=\sum_{i=1}^{N} \mathbf{S}(i)^{T} \hat{\mathcal{B}}^{-1} \mathbf{S}(i)+\boldsymbol{\Sigma}_{p}^{-1} \\
\hat{\boldsymbol{\theta}}=\hat{\boldsymbol{\theta}}_{0}-\left(\mathbf{M}+\boldsymbol{\Sigma}_{p}^{-1}\right)^{-1}\left(\frac{\partial J}{\partial \boldsymbol{\theta}}+\boldsymbol{\Sigma}_{p}^{-1}\left(\hat{\boldsymbol{\theta}}_{0}-\boldsymbol{\theta}_{p}\right)\right)
\end{gathered}
$$

where $\boldsymbol{\theta}_{p}$ and $\boldsymbol{\Sigma}_{p}$ are the prior parameter estimates and covariance matrix.

The last topic involves the accuracy of the estimated parameters, described by the Cramér-Rao bounds in Eq. (23). Output error is known to produce optimistic error bounds in the presence of colored innovations, which are not assumed in the theory but are routinely encountered with flight data, for example due to unmodeled dynamics. A method was developed for correcting these error bounds based on the coloring of the innovations..$^{25,1}$ This scenario is also true for filter error, where colored innovations are not assumed in the theory and lead to optimistic uncertainty bounds. This can be dangerous when using filter error because the match to the data can also be very good, which can give false confidence in the results. By extension of Ref. [25], the uncertainty bounds are corrected for the filter error problem by replacing Eq. (23) with

$$
\operatorname{cov}(\hat{\boldsymbol{\theta}}) \geq \mathbf{M}^{-1}\left[\sum_{i=1}^{N} \mathbf{S}(i)^{T} \hat{\mathcal{B}}^{-1}\left(\sum_{j=1}^{N} \hat{\mathcal{R}}_{v v}(i-j) \hat{\mathcal{B}}^{-1} \mathbf{S}(j)\right)\right] \mathbf{M}^{-1}
$$

where

$$
\hat{\mathcal{R}}_{v v}(k)=\frac{1}{N} \sum_{i=1}^{N-k} \boldsymbol{v}(i) \boldsymbol{v}(i+k)^{T}
$$

is the estimate of the discrete autocorrelation matrix for the innovations. These calculations are done at the end of the estimation process, after the final parameter estimates are identified.

\section{Results}

This section presents the modeling results. The test aircraft and its nonlinear simulation are first introduced. Then the excitation inputs and the dynamic model structure are presented. Lastly, simulation and flight test results are discussed. Results obtained using the proposed formulation of filter error were compared with results from output error using time-domain data and equation error using frequency-domain data, because these methods are popular and standard methods for aircraft parameter estimation. Uncertainties on all parameter estimates, for each method, have been corrected for colored residuals. 


\section{V.A. Test Aircraft and Simulation Model}

The test aircraft used is known as the T-2, and is shown in Fig. 2. The T-2 is a 5.5\% dynamically-scaled transport-type airplane used in the NASA Aviation Safety Program flight test capability called Airborne Subscale Transport Aircraft Research (AirSTAR). The aircraft is piloted after takeoff by a research pilot in a mobile control room using synthetic vision displays drawn from telemetry data and a database of the local terrain. Control deflection commands are generated from the research pilot and the ground-based flight control system. Computer-generated excitation inputs for system identification are added to these commands just before the position and rate limiters for the actuators.

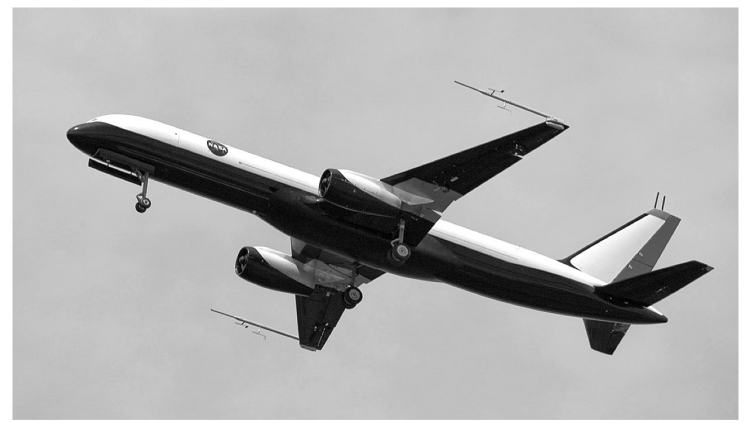

Figure 2. T-2 aircraft in flight (credit: NASA Langley Research Center)

The aircraft has 16 independent control surfaces, although these were moved as conventional elevator, aileron, and rudder surfaces in the present study. Measurements included body-axis translational accelerations and angular rates, estimated Euler angles, differential GPS position and velocity, air flow angles from vanes mounted on wingtip booms, static and dynamic pressure, fuel flow rate, and control surface deflections. First-order anti-aliasing filters with cut-off frequencies at $16 \mathrm{~Hz}$ were used before measurement digitization. Data were telemetered to the ground station at $200 \mathrm{~Hz}$, and downsampled to $50 \mathrm{~Hz}$ for analysis and modeling. For additional information about the aircraft and flight operations, see Ref. [26].

A high-fidelity, nonlinear, rigid-body, flight dynamics simulation of a test article similar to the T-2 aircraft, called the NASA Generic Transport Model (GTM), was previously developed using wind tunnel tests conducted at the NASA Langley Research Center. A $5.5 \%$ scaled test article was used in the 14- by 22-Foot Subsonic Tunnel under static and forced-oscillation testing to generate aerodynamic tables. These tables were augmented with data from rotary balance tests conducted using a $3.5 \%$ scaled wind tunnel test article in the 20-Foot Vertical Spin Tunnel. A polynomial-based aerodynamic model was then extracted from the data using multivariate orthogonal functions, ${ }^{27,1}$ expanded in the aircraft states and controls, and is implemented in the simulation. The GTM also includes two turbojet engines, the dynamics for which are modeled as first-order lags from the pilot throttle input to the thrust output.

\section{V.B. Maneuver Description and Model Structure}

Three sets of data are presented. The first set uses a linearized model of the GTM to simulate flight in light-to-moderate turbulence. The second set uses a $13 \mathrm{~s}$ maneuver of flight test data in light-to-moderate turbulence from T-2 Flight 41 . The third set uses five $13 \mathrm{~s}$ maneuvers of flight test data in severe turbulence from T-2 Flight 15. The simulation data was used to investigate algorithm performance in a controlled environment, but was devised to mimic conditions experienced in T-2 Flight 41. For each set of data, aircraft geometry and mass properties are listed in Table 1, and flight conditions are listed in Table 2.

Orthogonal phase-optimized multisine inputs were used to simultaneously excite the elevator, aileron, and rudder during straight and level trimmed flight. This type of input was devised at NASA Langley Research Center and has led to the efficient identification of many aircraft in a variety of unusual flight conditions including hypersonic, high sideslip, stall and post-stall flight, as well as normal flight conditions. ${ }^{18,1}$ The excitation used here lasted $10 \mathrm{~s}$ and contained frequencies between $0.2 \mathrm{~Hz}$ and $2.2 \mathrm{~Hz}$, staggered in 0.1 $\mathrm{Hz}$ increments. Control surface deflections were small enough to elicit responses appropriate for linear perturbation modeling, but large enough to produce good signal-to-noise ratios. The same input sequences were used in each set of data. 
The longitudinal short period model was investigated, although the data also contained excellent information content for lateral/directional modeling. The linear state-space approximation for these dynamics and measurements is ${ }^{1}$

$$
\begin{gathered}
{\left[\begin{array}{c}
\dot{\alpha} \\
\dot{q}
\end{array}\right]=\left[\begin{array}{cc}
-\frac{\bar{q} S}{m V} C_{L_{\alpha}} & 1-\frac{\bar{q} S}{\bar{m} V} \frac{\bar{c}}{2 V} C_{L_{q}} \\
\frac{\bar{q} S \bar{c}}{I_{y y}} C_{m_{\alpha}} & \frac{\bar{q} S \bar{c}}{I_{y y}} \frac{\bar{c}}{2 V} C_{m_{q}}
\end{array}\right]\left[\begin{array}{c}
\alpha \\
q
\end{array}\right]+\left[\begin{array}{c}
-\frac{\bar{q} S}{m V} C_{L_{\delta_{e}}} \\
\frac{\bar{q} S \bar{c}}{I_{y y}} C_{m_{\delta_{e}}}
\end{array}\right] \delta_{e}+\left[\begin{array}{c}
b_{\dot{\alpha}} \\
b_{\dot{q}}
\end{array}\right]+\left[\begin{array}{c}
w_{\alpha} \\
w_{q}
\end{array}\right]} \\
{\left[\begin{array}{c}
\alpha \\
q \\
a_{z}
\end{array}\right]=\left[\begin{array}{cc}
1 & 0 \\
0 & 1 \\
-\frac{\bar{q} S}{m g} C_{L_{\alpha}} & -\frac{\bar{q} S}{m g} \frac{\bar{c}}{2 V} C_{L_{q}}
\end{array}\right]\left[\begin{array}{c}
\alpha \\
q
\end{array}\right]+\left[\begin{array}{c}
0 \\
0 \\
-\frac{\bar{q} S}{m g} C_{L_{\delta_{e}}}
\end{array}\right] \delta_{e}+\left[\begin{array}{c}
0 \\
0 \\
b_{a_{z}}
\end{array}\right]+\left[\begin{array}{c}
v_{\alpha} \\
v_{q} \\
v_{a_{z}}
\end{array}\right]}
\end{gathered}
$$

Airspeed, dynamic pressure, and mass properties inside the matrices are constant nominal values at the initial trim condition. The states are not necessarily perturbation values because bias parameters have been included to account for initial conditions and steady-state values. Additive process noise and measurement noise have also been included. The unknown model parameters to be estimated are the nondimensional derivatives and the biases

$$
\boldsymbol{\theta}=\left[\begin{array}{lllllllll}
C_{L_{\alpha}} & C_{L_{q}} & C_{L_{\delta_{e}}} & C_{m_{\alpha}} & C_{m_{q}} & C_{m_{\delta_{e}}} & b_{\dot{\alpha}} & b_{\dot{q}} & b_{a_{z}}
\end{array}\right]^{T}
$$

although only the nondimensional derivatives are of interest. Estimating nondimensional derivatives removes the effects of known dependencies such as dynamic pressure.

\section{V.C. Simulation Results in Light-to-Moderate Turbulence}

A linearized model of the GTM was determined from the nonlinear simulation at the specified flight condition using central finite differences. The multisine inputs were used to excite the model. Process and measurement noise, having statistics extracted from the Flight 41 data in light-to-moderate turbulence, were used to corrupt the data. Additional colored noise in the frequency band $[0,3] \mathrm{Hz}$ and with amplitude equal to $5 \%$ of the root-mean-square value of each simulated output was added to simulate unmodeled dynamics. Although filter error can account for colored noise by augmenting the Kalman filter with a coloring filter, this was not done. The $\mathbf{R}$ used in the filter-error analysis was estimated using the spectral densities of the flight data between $10 \mathrm{~Hz}$ and $16 \mathrm{~Hz}$. This range was selected because experience with the T-2 showed that effects of turbulence attenuated by $7 \mathrm{~Hz}$, and the cutoff frequency for the anti-aliasing low-pass filters was set at $16 \mathrm{~Hz}$.

The simulation and analysis were performed 300 times, using unique noise sequences each time. One example run of the simulation data and the modeling result is shown in Fig. 3. The effects of turbulence are most evident during the quiet time before and after the excitation input. The measurements have high signal-to-noise ratios but also remain within the range for good linear modeling. The model outputs estimated using filter error had $R^{2}$ values above 0.99, making the measured and modeled outputs almost indistinguishable. The residuals had standard deviations less than $5 \%$ of the root-mean square of the signal variations. These metrics indicate an excellent modeling result.

On average, the proposed method estimated standard deviations of $\mathbf{R}$ within $8 \%$ and $\mathbf{Q}$ within $18 \%$ of the true values used in the simulation. Because the method relies on the supplied measurement noise covariance to estimate the parameters and the process noise covariance, some error in the estimation of $\mathbf{Q}$ can be attributed to error in $\mathbf{R}$. Again, output error and equation error absorb all the modeling error into either the $\mathbf{R}$ or $\mathbf{Q}$ matrix, respectively, and it is not expected that they match the true values. In terms of estimating noise statistics, the filter-error method proposed here estimated both sources, and did so more accurately, than either output error or equation error alone. The achieved level of accuracy is good, considering the presence of colored noise in the data and the robustness of the Kalman filter to modeling errors. The output error software typically converged within 21 iterations, whereas filter-error software typically used 24 iterations. This increase in computation time is small, especially compared to the increased accuracy gained from using filter error.

The parameter estimation results for all 300 simulation runs are summarized in Fig. 4. The marker locations indicate the mean parameter estimates. The left bars show the estimated $2 \sigma$ uncertainty in the model parameters using Eq. (30), and the right bars show the $2 \sigma$ scatter in the estimates from repeated 

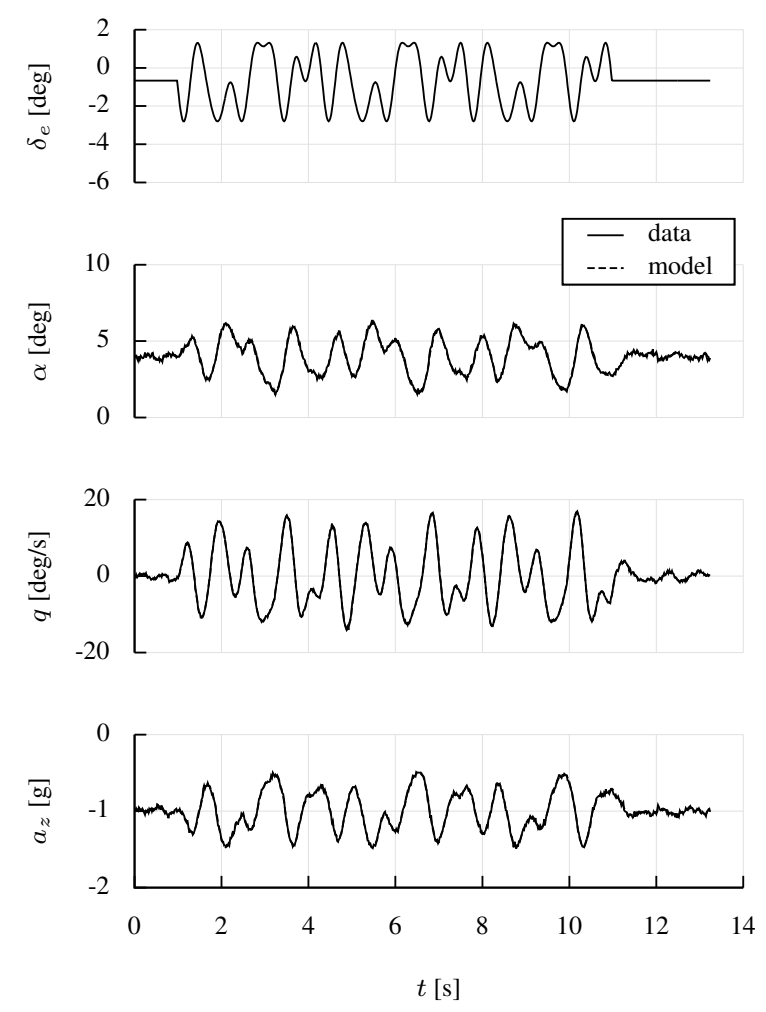

Figure 3. Measurements and model fit using filter error, Monte Carlo run 300

experiments. Equal bars indicate an accurate prediction of the parameter uncertainty. All estimates are relatively accurate; however, errors are evident because process noise biases output error, measurement noise biases equation error, and unmodeled dynamics biases all three methods. In general, the filter error estimate had the most accurate parameter estimates. In addition, the filter error estimates had the smallest error bounds, was the only method that always produced estimates within statistical agreement of the true parameters, and was the method that most closely matched predicted uncertainty with observed scatter in the parameter estimates.

Estimates of $C_{m_{q}}$ for a few example Monte Carlo runs are shown in Fig. 5. The pitch damping derivative is often one of the harder model parameters to accurately identify. However, these results corroborate those in Fig. 4 and demonstrate that filter error consistently had more accurate estimates and smaller uncertainties which were also in statistical agreement with the true value. Estimates using output error and equation error exhibited more variation, larger error bounds, and degraded statistical agreement with the true value.

The sensitivity of the filter error results to the estimated $\mathbf{R}$ was investigated. Results for $C_{m_{q}}$ are shown in Fig. 6 for varying levels of error. For $\mathbf{R}$ too low, the Kalman filter weights the measured output data over the model output, and the parameter estimates began to diverge from their true values, until estimates no longer converged. This was also accompanied by an increased number of iterations needed for convergence. For $\mathbf{R}$ too high, the Kalman filter weights the model output over the measured output data, and the estimates of $C_{m_{q}}$ were relatively insensitive until very large errors in $\mathbf{R}$, although other parameters and indicators changed. A $40 \%$ error in the noise standard deviation was tolerated before the parameter estimates were no longer in statistical agreement with the true parameters. In practice, however, error margins for $\mathbf{R}$ will decrease due to uncertainty in the model structure.

\section{V.D. Flight Test Results in Light-to-Moderate Turbulence}

The T-2 aircraft was excited using a multisine input, starting from trimmed straight and level flight in light-to-moderate turbulence. The measured flight data, as well as the model fit using filter error, is shown in Fig. 7. The elevator waveform is very similar to that shown in Fig. 3. The small difference in starting and ending values is due to the pilot applying a small amount of back-pressure to the stick during the maneuver. The higher-frequency motion of the control surfaces are attenuated by the flight control system and actuator 

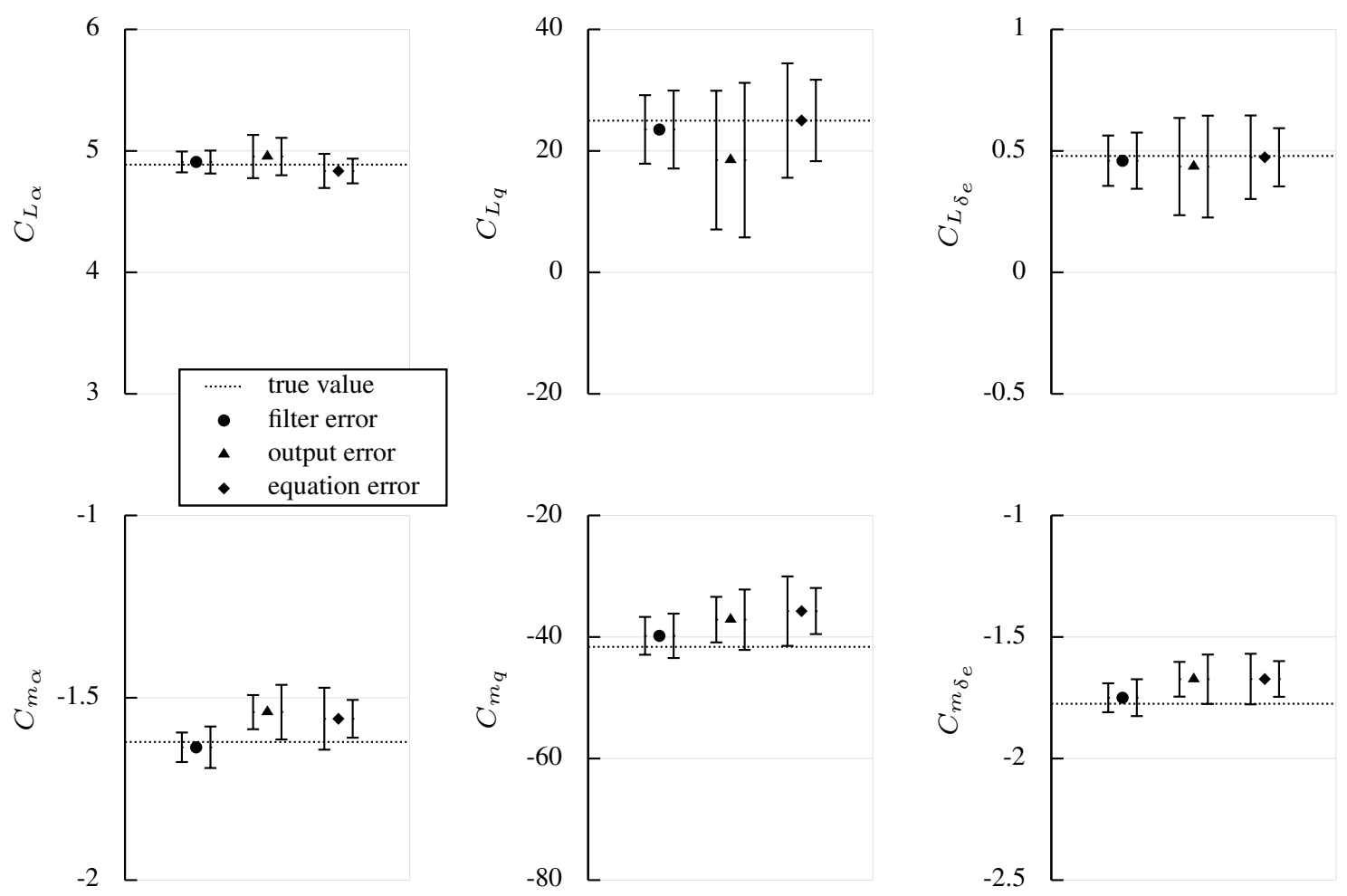

Figure 4. Parameter estimation results for 300 Monte Carlo runs: marker $=$ mean parameter estimate, left bar $=2 \sigma$ uncertainty, right bar $=2 \sigma$ scatter

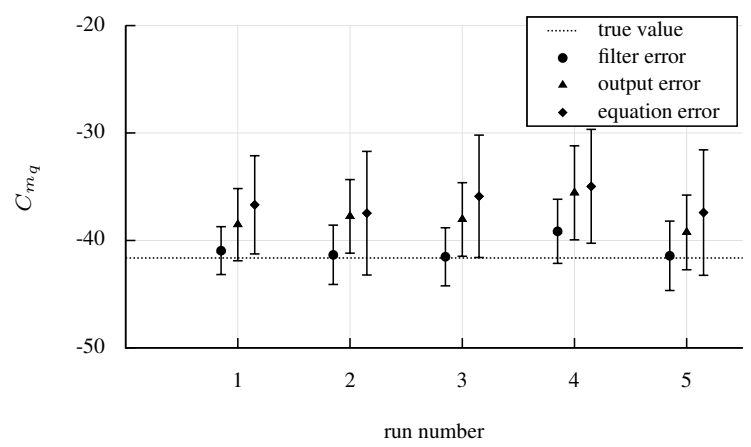

Figure 5. Example parameter estimates and $2 \sigma$ uncertainties, Monte Carlo runs 1-5

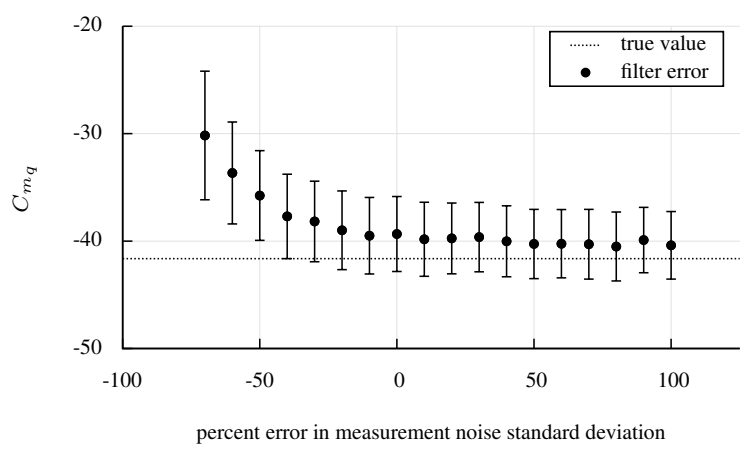

Figure 6. Sensitivity of parameter estimates to measurement noise error 
dynamics. The measured responses are also very similar, and again have high signal-to-noise ratios and good ranges for linear modeling. Modeled outputs using filter error had an $R^{2}$ value of 0.98 , again indicating an excellent match.
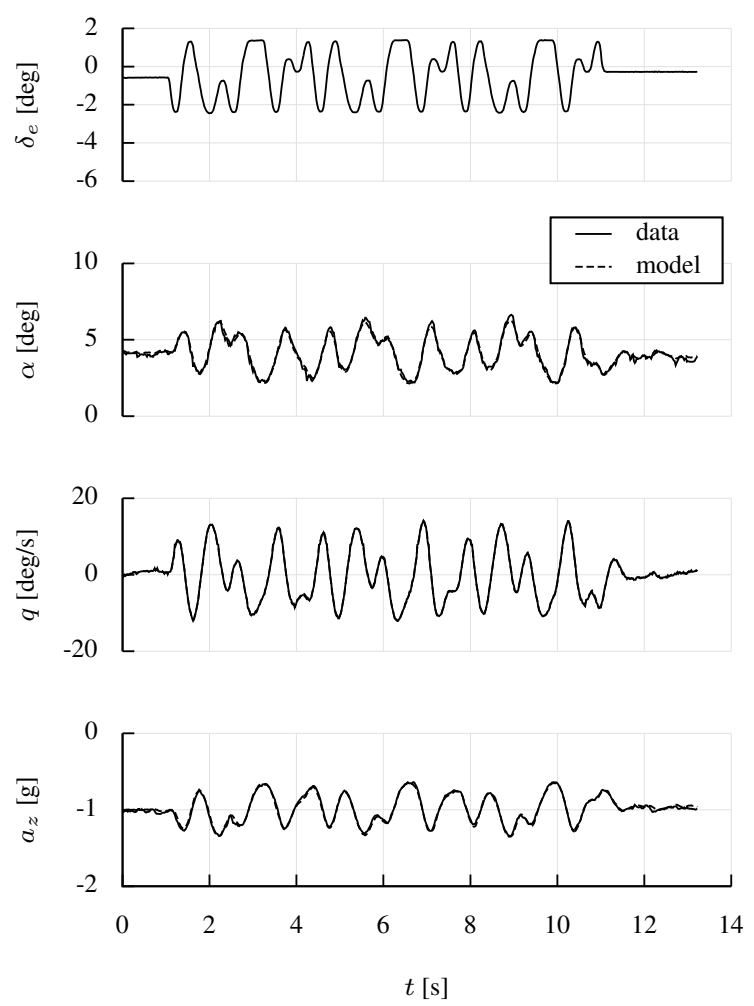

Figure 7. Measurements and model fit using filter error, T-2 Flight 41

The estimated short-period parameters are shown in Fig. 8. The parameter estimates differed from those in Fig. 4 because those were computed from wind tunnel data and these were estimated from flight test data. The estimated parameters were consistent between the three methods. The output-error results predicted the lowest error bounds, but these results can be biased because of the turbulence. The equation-error results had the highest error bounds. The filter-error results represent a middle ground and were expected to be the most accurate results.

The sensitivity of the model outputs to model parameter variations was investigated. Figure 9 shows the change in the pitch rate time history due to a change in the pitch damping derivative, for both output error and filter error. This plot confirms that integration using output error is about an order of magnitude more sensitive than Kalman filtering using filter error. Output error used 18 iterations to converge in this case, whereas filter error used 36 iterations. The decreased sensitivity of filter error resulted in more computation time than output error, but attained more accurate results. Balancing computation time versus better accuracy and uncertainty information is a trade-off that the analyst must make when choosing between output error and filter error for data collected in modest levels of turbulence.

\section{V.E. Flight Test Results in Severe Turbulence}

The T-2 aircraft was excited using a multisine input, starting from trimmed straight and level flight in severe turbulence. Five repeated maneuvers were considered. Measurements and model outputs using filter error are shown in Fig. 10 for Maneuver B. The elevator time histories differ slightly from those in Fig. 7 because the pilot provided more input to keep the aircraft near the nominal flight condition. Measurements of the aircraft response are no longer smooth and now appear jagged, due to the effects of turbulence. Higher angles of attack, pitch rate, and normal acceleration were reached, which could degrade the linearity assumptions. However, filter error still produced an excellent fit to the measured data, having $R^{2}$ values above 0.98 .

Figure 11 presents an analysis of the angle of attack innovations for Maneuver B using filter error. The 

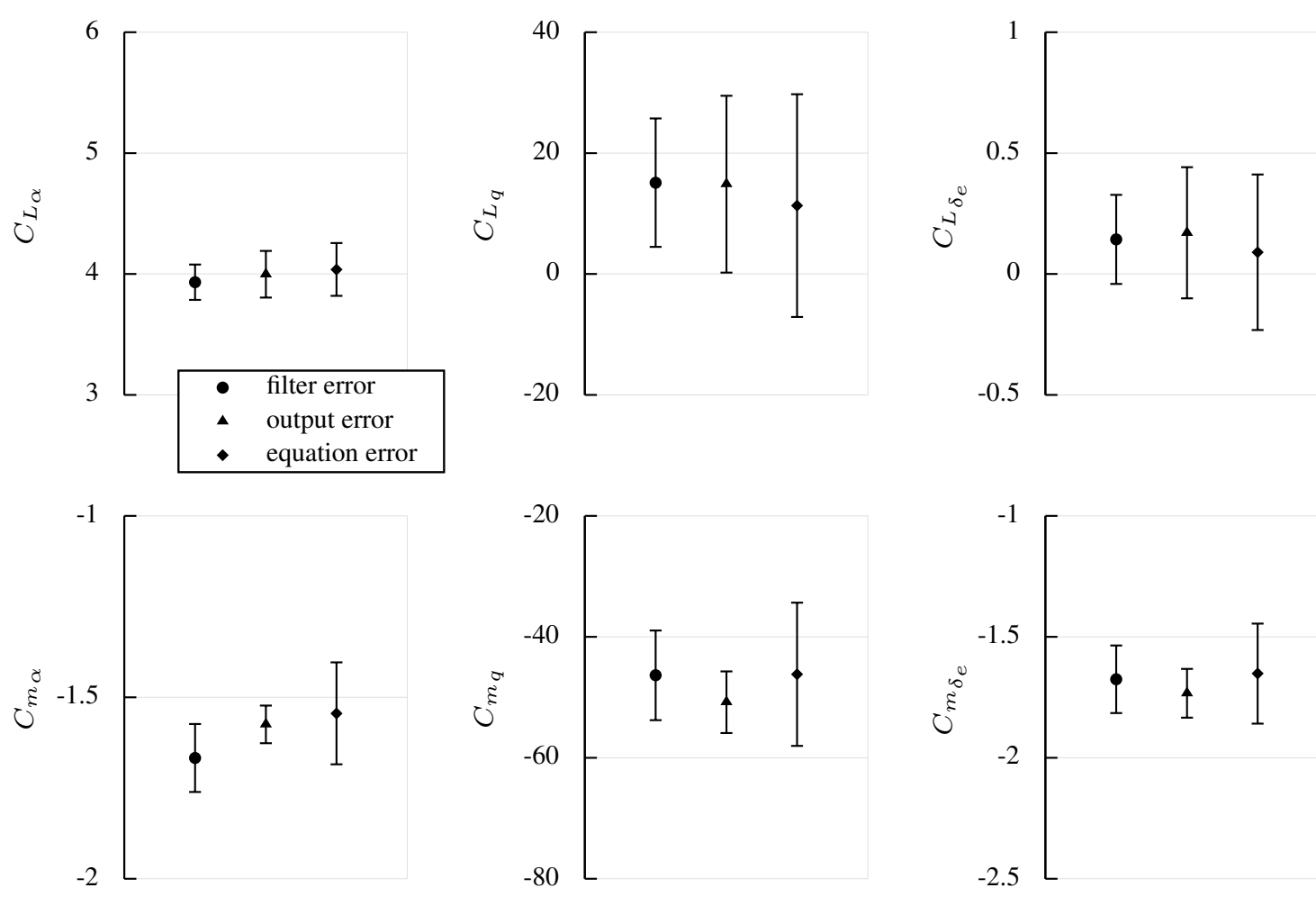

Figure 8. Parameter estimates and $2 \sigma$ uncertainties, T-2 Flight 41

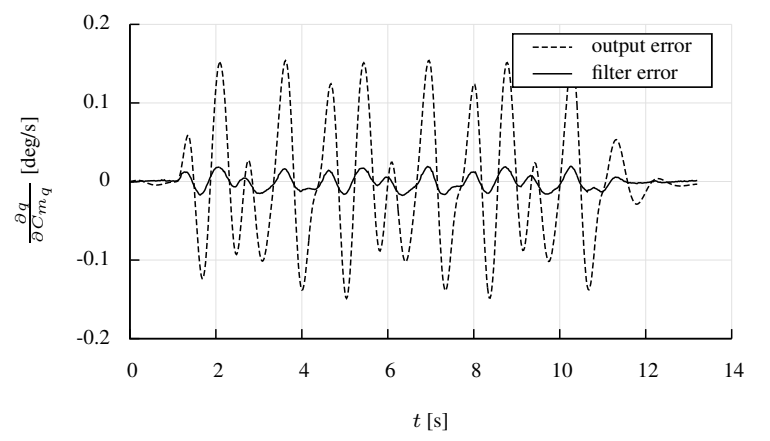

Figure 9. Pitch rate sensitivity to pitch damping, T-2 Flight 14 

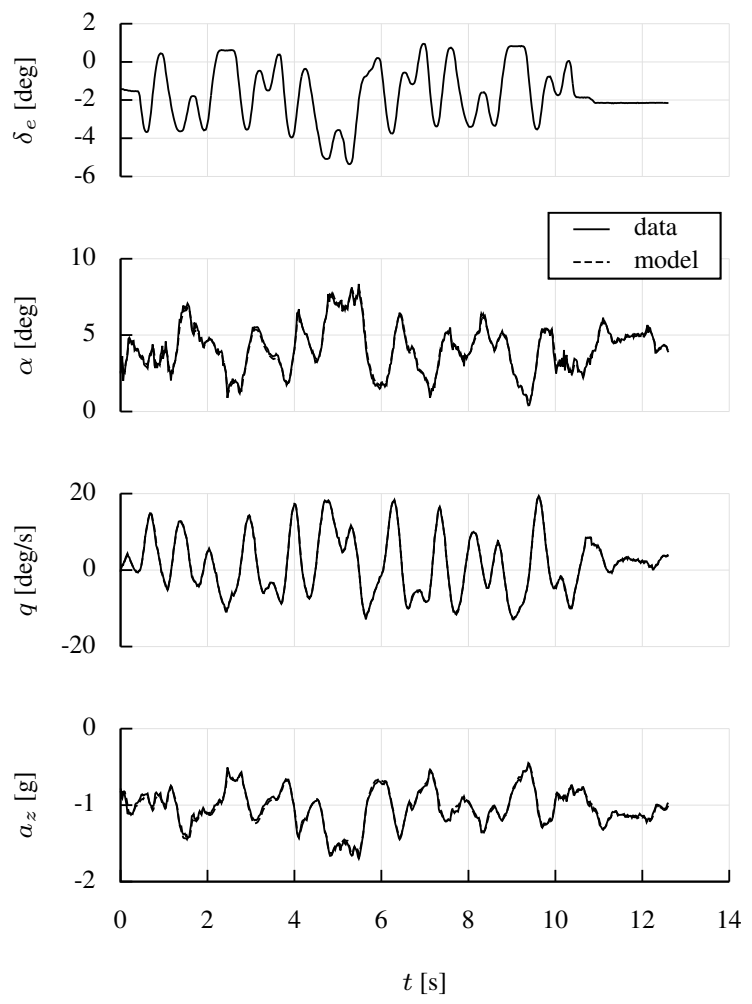

Figure 10. Measurements and model fit using filter error, T-2 Flight 15 Maneuver B

assumption of normally-distributed disturbances can be visually inspected using Fig. 11(a), which shows the probability density of the innovations and an ideal Gaussian curve with the same mean and covariance. These residuals approximate a Gaussian distribution well and have $R^{2}=0.95$, which indicates that the Gaussian noise assumption is valid. The probability densities for the output error and equation error results had much larger standard deviations, multi-modal distributions, lower fits with an idealized Gaussian distribution, although this is conceded by the simplifying assumptions in these methods.

The assumption of white noise can also be visually inspected using Fig. 11(b), which shows the innovation autocorrelation and the $2 \sigma$ confidence interval (computed as $\pm 2 \hat{\mathcal{R}}_{v v}(0) / \sqrt{N}$ ). Because this plot contains a sharp peak at zero lag and small values within the uncertainty band for most other lags, the white-noise assumption is valid. The autocorrelations for the output-error and equation-error results were an order of magnitude larger and had more data outside the confidence intervals. This indicated that the innovations were larger and had significant coloring.

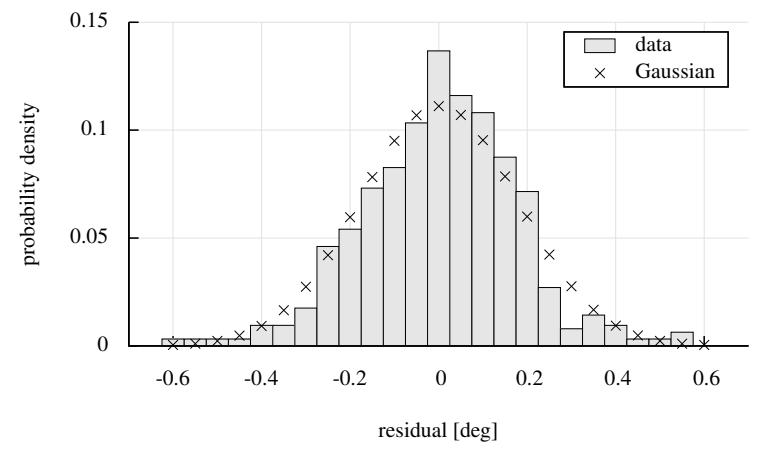

(a) test for normality

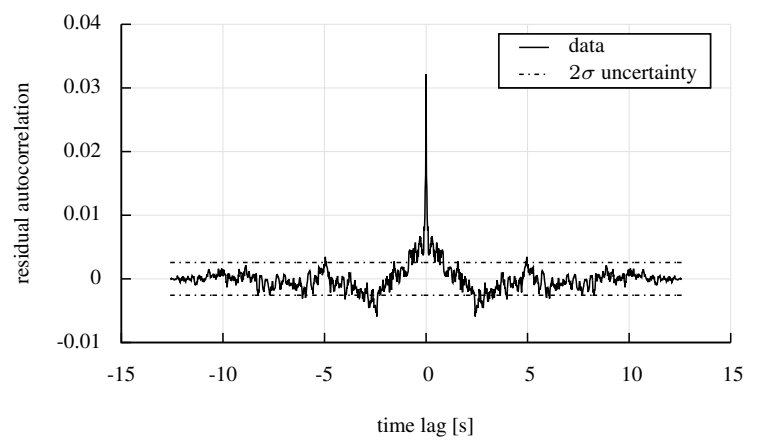

(b) test for whiteness

Figure 11. Angle of attack modeling diagnostics using filter error, T-2 Flight 15 Maneuver B

Figure 12 shows the modeling results for T-2 Flight 15 . The output-error parameters exhibited a large 
amount of scatter and uncertainty, especially in the damping derivatives, due to the high level of turbulence. The filter-error and equation-error results were much more consistent and accurate. The equation-error estimates of $C_{L_{\alpha}}$ and $C_{m_{\alpha}}$ exhibited a shift from the Flight 41 estimates. This shift was also seen in Ref. [17] and is expected to be due to an unsteady dependence on the angle of attack. ${ }^{28}$ Because filter error includes a more general and accurate characterization of the noise and system dynamics, parameter estimates from Flight 15 data in severe turbulence were more consistent with those from Flight 41 data in light-to-moderate turbulence. For the remaining stability and control derivatives, while some scatter and variability was seen in the estimates between maneuvers, the new formulation of filter error produced similar results as frequency domain equation error, which is known to produce excellent modeling results.
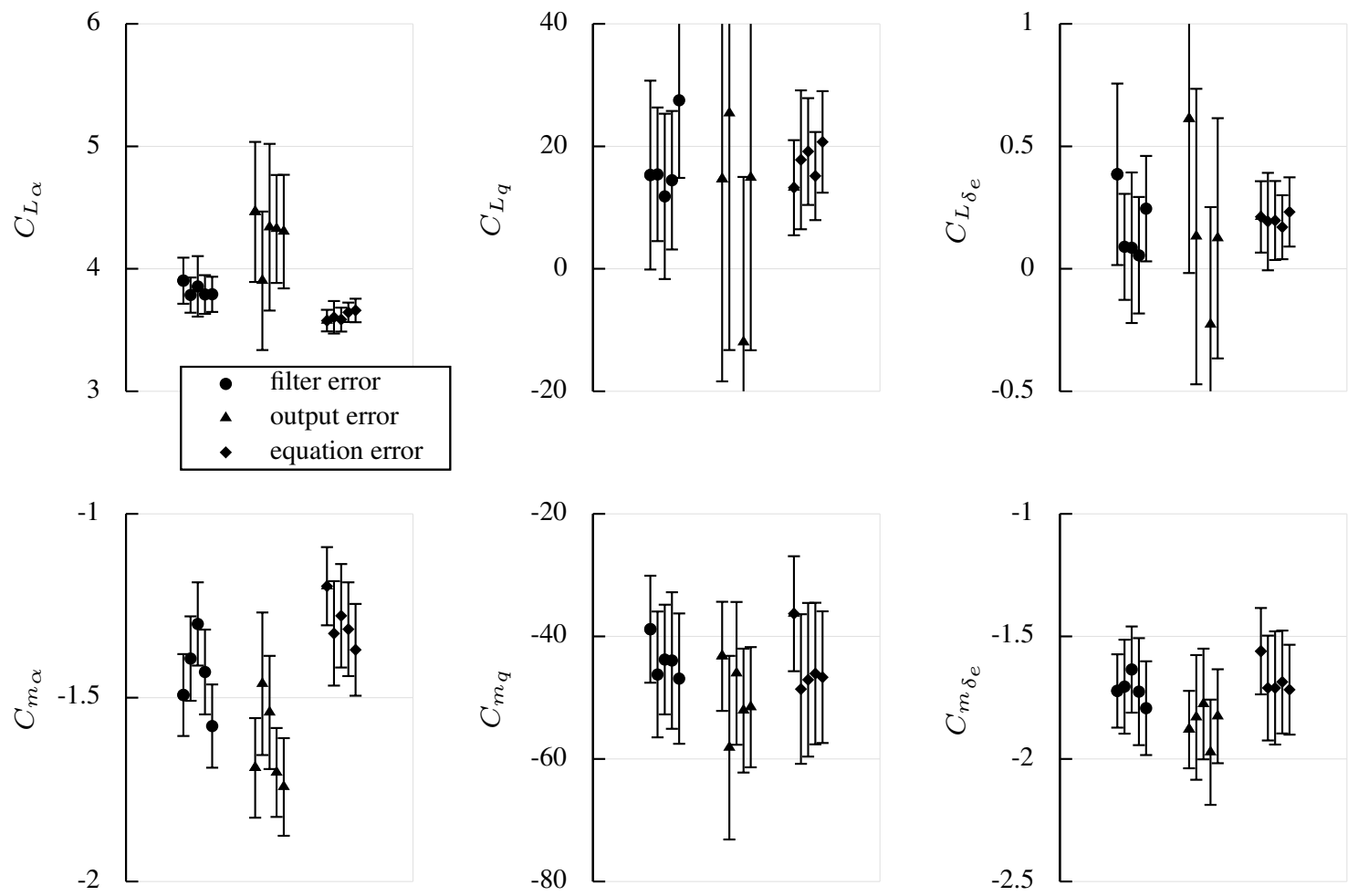

Figure 12. Parameter estimates and $2 \sigma$ uncertainties, T-2 Flight 15

Table 3 lists the parameter estimates and their uncertainties for T-2 flights 41 and 15 using filter error. The results here are similar and within statistical agreement of each other, and are similar to those estimated previously in Ref. [17] using equation error in the frequency domain. The uncertainties are lower for the Flight 15 results because more maneuvers were used in the estimation. The fact that consistent results were attained using filter error with flight test data under different levels of turbulence demonstrates that this implementation of filter error produced excellent modeling results.

Table 4 displays the estimated statistics for the process noise and measurement noise using T-2 flights 41 and 15. The measurement noise estimates were consistent between these two flights, which was expected because instrumentation did not change. The amount of process noise increased from Flight 41 to Flight 15, approximately doubling the diagonal components, which was expected from the higher turbulence rating. Similar to the metric developed in Ref. [17], turbulence levels can now be correlated with values for $\mathbf{Q}$.

\section{Conclusions}

A new formulation of the filter error method for estimating aerodynamic parameters in turbulence was presented. The method is designed for batch analysis, after all the flight data have been collected. It is applicable to general nonlinear systems, and any method of state estimation may be used. Given an estimate of the measurement noise covariance, a relaxation technique is used to alternately update the model parameters and the process noise covariance. This process is akin to that used in the traditional output-error algorithm, and is familiar to engineers and straightforward to apply. No ad hoc adjustments 
of tuning parameters are used, so that the method can be applied consistently and accurately by different analysts. Prior information from other analyses and experiments can be supplied to the estimator in a Bayesian framework. A correction was included to account for colored residuals, which are not assumed in the theory. The formulation was implemented as MATLAB ${ }^{\circledR}$ software and runs using reasonable amounts of computation time, only slightly more than conventional output error.

The method was applied to simulation data to understand the nature of the results. The method was also applied to flight test data in light-to-moderate turbulence and severe turbulence. Results were consistent between flights and performed better than time domain output error, and at least as good as frequency domain equation error. No convergence or identifiability issues were encountered. Modeling assumptions were demonstrated to be valid. Computed parameter uncertainty measures were shown to be consistent with scatter in the parameter estimates from repeated maneuvers. Fits of the model outputs to the measured output data were excellent.

Although developed for general nonlinear dynamic systems, the discussion presented here was demonstrated using a linear dynamic model as a special case. This was done because aircraft parameter estimation routinely is used to estimate stability and control derivatives in linear models. Since linear models are often appropriate and noise statistics may be assumed stationary during standard maneuvers for system identification, a steady-state Kalman filter was also used. Nonlinear models and state estimators may however be used in their place.

Knowledge of the measurement noise statistics is the only extra information needed by the analyst in this formulation of filter error. Because measurement noise has a clear physical interpretation and can be modeled well, and because there are several methods for accurately determining the measurement noise, this step is straightforward and not difficult to do. There is also some margin for error in this step, depending on the level of accuracy in the model structure. The benefit of this approach is that the estimator is relieved from having to estimate measurement noise, which makes the overall nonlinear estimation problem better conditioned and more accurate. In effect, the number of unknowns to be estimated is reduced because the measurement noise can be accurately estimated separately by other means.

It was demonstrated that this filter error implementation can provide results superior to time domain output error, and at least as good as frequency domain equation error, when turbulence and measurement noise is present in the data. Unless both noise sources are substantial, however, the simpler methods should be preferred, to reduce computation time and analyst effort. However, if significant process noise exists, or all of the explanatory variables cannot be measured accurately or reconstructed, then filter error must be used. In addition to aerodynamic modeling in turbulence, other application examples include unsteady aerodynamic modeling, where unobservable aerodynamic states are included in the model, linearized modeling in significantly nonlinear regimes, or flexible aircraft modeling, where modal states may not be available. In these cases, which are becoming more prevalent in flight testing, filter error can produce more accurate modeling results, in addition to providing more detailed information about the amplitude and nature of the noise sources.

\section{Acknowledgments}

The efforts of the AirSTAR flight test team at NASA Langley in building and testing the T-2 aircraft and associated systems, carefully calibrating the instrumentation, and carrying out the flight operations to collect the high-quality flight data used in this study, are gratefully acknowledged. This research was funded by the NASA Subsonic Fixed-Wing project. 


\section{Tables}

Table 1. Aircraft geometry and nominal mass properties

\begin{tabular}{ccccc}
\hline \hline Parameter & GTM & T-2 Flight 41 & T-2 Flight 15 & Unit \\
\hline $\bar{c}$ & 0.915 & 0.915 & 0.915 & $\mathrm{ft}$ \\
$S$ & 5.902 & 5.902 & 5.902 & $\mathrm{ft}^{2}$ \\
$m$ & 1.639 & 1.639 & 1.646 & slug \\
$I_{y y}$ & 4.651 & 4.651 & 4.652 & slug.ft \\
\hline \hline
\end{tabular}

Table 2. Nominal flight conditions

\begin{tabular}{ccccc}
\hline \hline Parameter & GTM & T-2 Flight 41 & T-2 Flight 15 & Unit \\
\hline$V$ & 140.7 & 139.1 & 136.0 & $\mathrm{ft} / \mathrm{s}$ \\
$\alpha$ & 4.000 & 4.077 & 3.893 & $\mathrm{deg}$ \\
$h$ & 1226 & 1227 & 1467 & $\mathrm{ft}$ \\
$\delta_{t}$ & 0.320 & 0.290 & 0.315 & - \\
\hline \hline
\end{tabular}

Table 3. Parameter estimates and uncertainties identified from T-2 flight data using filter error

\begin{tabular}{ccc}
\hline \hline Parameter & T-2 Flight 41 & T-2 Flight 15 \\
$\theta$ & $\hat{\theta} \pm \sigma(\hat{\theta})$ & $\hat{\theta} \pm \sigma(\hat{\theta})$ \\
\hline$C_{L_{\alpha}}$ & $+3.933 \pm 0.073$ & $+3.828 \pm 0.036$ \\
$C_{L_{q}}$ & $+15.11 \pm 5.319$ & $+16.39 \pm 2.719$ \\
$C_{L_{\delta_{e}}}$ & $+0.143 \pm 0.092$ & $+0.125 \pm 0.054$ \\
$C_{m_{\alpha}}$ & $-1.667 \pm 0.047$ & $-1.437 \pm 0.025$ \\
$C_{m_{q}}$ & $-46.36 \pm 3.712$ & $-44.76 \pm 2.169$ \\
$C_{m_{\delta_{e}}}$ & $-1.676 \pm 0.070$ & $-1.722 \pm 0.040$ \\
\hline \hline
\end{tabular}

Table 4. Estimated measurement and process noise levels from T-2 flight data using filter error

\begin{tabular}{cccc}
\hline \hline Parameter & T-2 Flight 41 & T-2 Flight 15 & Unit \\
\hline std $v_{\alpha}$ & 0.199 & 0.232 & $\mathrm{deg}$ \\
std $v_{q}$ & 0.260 & 0.274 & $\mathrm{deg} / \mathrm{s}$ \\
std $v_{a_{z}}$ & 0.046 & 0.045 & $\mathrm{~g}$ \\
std $w_{\alpha \alpha}$ & 0.052 & 0.099 & $\mathrm{deg} / \mathrm{s}$ \\
std $w_{\alpha q}$ & 0.044 & 0.063 & $\mathrm{deg} / \mathrm{s}^{3 / 2}$ \\
std $w_{q q}$ & 0.152 & 0.294 & $\mathrm{deg} / \mathrm{s}^{2}$ \\
\hline \hline
\end{tabular}




\section{References}

${ }^{1}$ Klein, V. and Morelli, E., Aircraft System Identification: Theory and Practice, AIAA Education Series, AIAA, Reston, VA, 2006 .

${ }^{2}$ Maine, R. and Iliff, K., "Identification of Dynamic Systems: Theory and Formulation," Tech. Rep. RP-1138, NASA, Edwards, CA, February 1985.

${ }^{3}$ Morelli, E. and Klein, V., "Application of System Identification to Aircraft at NASA Langley Research Center," Journal of Aircraft, Vol. 42, No. 1, January-February 2005, pp. 12-25.

${ }^{4}$ Wang, K. and Iliff, K., "Retrospective and Recent Examples of Aircraft Parameter Identification at NASA Dryden Flight Research Center," Journal of Aircraft, Vol. 41, No. 4, July-August 2004, pp. 752-764.

${ }^{5}$ Morelli, E., "Practical Aspects of the Equation-Error Method for Aircraft Parameter Estimation," No. 2006-6144 in Atmospheric Flight Mechanics Conference, AIAA, Keystone, CO, August 2006.

${ }^{6}$ Maine, R. and Iliff, K., "Application of Parameter Estimation to Aircraft Stability and Control: The Output-Error Approach," Tech. Rep. RP-1168, NASA, Edwards, CA, June 1986.

${ }^{7}$ Morelli, E., "System IDentification Programs for AirCraft (SIDPAC)," No. 2002-4704 in Atmospheric Flight Mechanics Conference, AIAA, Monterey, CA, August 2002.

${ }^{8}$ Gelb, A., editor, Applied Optimal Estimation, The MIT Press, Cambridge, MA, 1974.

${ }^{9}$ Jategaonkar, R. and Plaetschke, E., "Algorithms for Aircraft Parameter Estimation Accounting for Process and Measurement Noise," Journal of Aircraft, Vol. 26, No. 4, April 1989, pp. 360-372.

${ }^{10}$ Chowdhary, G. and Jategaonkar, R., "Aerodynamic Parameter Estimation from Flight Data Applying Extended and Unscented Kalman Filter," Aerospace Science and Technology, Vol. 14, No. 2, March 2010, pp. 106-117.

${ }^{11}$ Maine, R. and Iliff, K., "Formulation and Implementation of a Practical Algorithm for Parameter Estimation with Process and Measurement Noise," SIAM Journal on Applied Mathematics, Vol. 41, No. 3, December 1981, pp. 558-579.

${ }^{12}$ Morelli, E., "Estimating Noise Characteristics from Flight Test Data Using Optimal Fourier Smoothing," Journal of Aircraft, Vol. 32, No. 4, July-August 1995, pp. 689-695.

${ }^{13}$ Özger, E., "Introducing a Combined Equation/Output Error Approach in Parameter Estimation," No. 2010-0034 in Aerospace Sciences Meeting, AIAA, Orlando, FL, January 2010.

${ }^{14}$ Murray, J. and Maine, R., "pEst Version 2.1 User's Manual," Tech. Rep. TM-88280, NASA, Edwards, CA, September 1987.

${ }^{15}$ Martos, B. and Morelli, E., "Using Indirect Turbulence Measurements for Real-Time Parameter Estimation in Turbulent Air," No. 2012-4651 in Atmospheric Flight Mechanics Conference, AIAA, Minneapolis, MN, August 2012.

${ }^{16}$ Morelli, E., "Real-Time Parameter Estimation in the Frequency Domain," Journal of Guidance, Control, and Dynamics, Vol. 23, No. 5, September-October 2000, pp. 812-818.

${ }^{17}$ Morelli, E. and Cunningham, K., "Aircraft Dynamic Modeling in Turbulence," Atmospheric Flight Mechanics Conference, AIAA, Minneapolis, MN, August 2012.

${ }^{18}$ Morelli, E., "Flight Test Maneuvers for Efficient Aerodynamic Modeling," Journal of Aircraft, Vol. 49, No. 6, NovemberDecember 2012, pp. 1857-1867.

${ }^{19}$ Schultz, G., "Maximum Likelihood Identification Using Kalman Filtering Least-Squares Estimation: A Comparison for the Estimation of Stability Derivatives Considering Gust Disturbances," Tech. Rep. DLR-FB 75-94, DLR, February 1975.

${ }^{20}$ Iliff, K., Identification and Stochastic Control with Application to Flight Control in Turbulence, Ph.D. thesis, University of California, Los Angeles, Los Angeles, CA, May 1973.

${ }^{21}$ Maine, R. and Iliff, K., "User's Manual for MMLE3, A General FORTRAN Program for Maximum Likelihood Parameter Estimation," Tech. Rep. TP-1563, NASA, Edwards, CA, November 1980.

${ }^{22}$ Jategaonkar, R., "ESTIMA - An Integrated Software Tool for Nonlinear Parameter Estimation," Aerospace Science and Technology, Vol. 6, No. 8, December 2002, pp. 565-578.

${ }^{23}$ Foster, G., "The Identification of Aircraft Stability and Control Parameters in Turbulence," Tech. Rep. TR-83025, RAE, March 1983.

${ }^{24}$ Maine, R. and Murray, J., "Application of Parameter Estimation to Highly Unstable Aircraft," Tech. Rep. TM-88266, NASA, Edwards, CA, August 1986.

${ }^{25}$ Morelli, E. and Klein, V., "Accuracy of Aerodynamic Model Parameters Estimated from Flight Test Data," Journal of Guidance, Control, and Dynamics, Vol. 20, No. 1, January-February 1997, pp. 74-80.

${ }^{26}$ Jordan, T., Foster, J., Bailey, R., and Belcastro, C., "AirSTAR: A UAV Platform for Flight Dynamics and Control System Testing," No. 2006-3307 in Aerodynamic Measurement Technology and Ground Testing Conference, AIAA, San Francisco, CA, June 2006.

${ }^{27}$ Morelli, E., "Global Nonlinear Aerodynamic Modeling Using Multivariate Orthogonal Functions," Journal of Aircraft, Vol. 32, No. 2, March-April 1995, pp. 270-277.

${ }^{28}$ Klein, V. and Noderer, K., "Modeling of Aircraft Unsteady Aerodynamic Characteristics: Part 3 — Parameters Estimated from Flight Data," Tech. Rep. TM-110259, NASA, Hampton, VA, May 1996. 